\title{
Research on Problems and Countermeasures of Doctor-Patient Communication from the Perspective of Interpersonal Communication
}

\author{
Chen $\mathrm{LI}^{1, a}$, Li WANG ${ }^{2, b, *}$ \\ ${ }^{1}$ No.2006, Xiyuan Avenue, West Hi-tech Zone, Chengdu, Sichuan, P.R. China \\ ${ }^{2}$ No.2006, Xiyuan Avenue, West Hi-tech Zone, Chengdu, Sichuan, P.R. China \\ 649098611@qq.com
}

\begin{abstract}
Keywords: Interpersonal Communication, Doctor-patient Communication, Health Communication, Cognitive Dissonance.
\end{abstract}

\begin{abstract}
In this paper, we discuss problems in the process of doctor-patient communication through observational and in-depth interviewing method, from the perspective of interpersonal communication. The study find that cognitive dissonance, a lack of communication opportunities and interpersonal skills in medical workers, as well as negative emotions caused by them are the aim barriers for communication between each other.in view of this finding, this paper put forward some suggestions, such as using new technology to innovate doctor-patient communication form, paying attention to psychological and emotional interaction.
\end{abstract}

\section{Introduction}

This paper is based on the perspective of one-to-one interpersonal communication, through the actual observation of the hospital environment and in-depth interviews with doctors and patients, analyzing the content of information and emotional communication between doctors and patients, to explore the communication barriers between doctors and patients, striving to get a reasonable and effective means of interpersonal communication.

\section{Research Status and Thinking}

The early 80 s of last century, China's academic community began to study the doctor-patient relationship. With the continuous progress of reform and opening up, China's medical system and the relevant management mechanism is also a step by step to improve, but the doctor-patient relationship is more complex, medical disputes are frequent, research of doctor-patient relationship is also deepening.

\section{Research Status}

CNKI, Wanfang database are currently more authoritative database, because of its significant representation, literature from which can represent a field of research status quo. The author uses "interpersonal communication + doctor-patient communication" as the search term, searching the 2006 - 2016 literature, a total of 46 related literature, which contains four master thesis. At present, the research status of doctor-patient communication in China is mainly focused on influencing factors, countermeasures, cases and so on.

\section{Study on Influencing Factors in the Process of Doctor-patient Communication}

In the internal factors that affect doctor-patient communication, Zhang zili put forward the concept of personalized communication, which is not only relevant to patient's demographic properties, but also with the patient's stored position, behavior patterns and beliefs. From sociological and psychological perspective, Han Qing (2014) analyze that comprehensive quality people owning is higher, the intervention of diagnosis process is stronger, the more questions. But if people have doubts, they will be more compliant and submitted to sense of professional sacredness. The mass media coverage of the doctor-patient contents is an important external factor affecting the doctor- 
patient communication. Peng Man (2007) found that China's patient image coverage in newspaper is almost negative.

Through comparative analysis of doctor-patient relationship report on "Xinmin Evening News" and "Morning News", Shi Qiuling (2013) concluded that media pay attention to health communication, be less concern about doctor-patient relationship, especially medical disputes report. Li Xuefei (2014) pointed out that media agenda set up improperly, unexpected media positions in the wavering, as well as one-sided coverage on doctor's image and other issues.

\section{A Study on the Countermeasures of Doctor - Patient Communication}

Yuan weiwei (2013) proposed improving rules and regulations for doctor-patient communication, put doctor-patient communication requirements integrated into hospital medical quality management system and evaluation system of health administrative departments. Han Xueying and Zhang han (2015) proposed tripple models of doctor-patient communication: active - passive, guidance - cooperation and two-way participation. HOU Sheng-tian, ZHANG Yong-kang (2014) proposed the "6S extension of doctor-patient communication model", which is divided into six stages: preparation, integration, interaction, education, negotiation and maintenance. Wang Yueping, Lu Fan (2009) constructed the external harmony evaluation model of medical institutions, including the professional ethics, standard service, medical environment, technical level, medical expenses, complaints, expectations, image, overall evaluation. In addition to countermeasures in system model, there are scholars from the sociology, psychology, management and other aspects of the consultation giving advice on doctor-patient communication, such as Chen Li, Wang Yongan (2008) try to dig out patient personality psychology form and pattern from the perspective of patient's humanistic care. Feng Junqiang (2008) suggested that medical institutions alloctae human resources rationally, medical staff pay attention to its skills in verbal and nonverbal communication.

\section{Case Study of Doctor - Patient Communication}

The study of this part refers to general phenomenon of doctor-patient communication disorder from a doctor-patient disorder or medical disturbance. By investigate a large-scale public hospital, Wang furong and other people (2012) find out that the major reason resulting to doctor-patient communication barriers includes lack of faith between doctors and patients, ignoring patients' communication needs, interpersonal skills absence, humanistic quality being not high and so on. Dai yuanguang, Han ruixia (2012) discovered that the general evaluation of the current doctorpatient relationship in China was neutral, the evaluation of medical environment such as medical convenience was high and the problems encountered in the process of medical treatment were mostly attributed to "resources shortage" "poor communication" "lack of trust". They finished this investigation through sampling survey method in Shandong、Shanghai、Zhejiang and Xinjiang, which consisted of a total of 700 people. By cluster random sampling between medical staff and patients in Beijing, Wuhan, Nanning, Kaili, Aksu. Leichang and Zhangsiyuan (2009) conformed that doctor-patient diaorder exert greater influence on the side of hospital.

\section{Problem Raising and Research Method}

\section{Statement of Problem}

Results above established a solid condition for further research, but there are also deficiencies, such as doctor-patient communication influence factor, involving social insurance, medical market, social science and technology culture, pressure in doctors and patients and so on. Under the new social circumstance, what are the barriers in doctor-patient communication? Can the progress of science and technology offering assistance for growing doctor-patient dispute? What are the advice in the perspective of interpersonal communication? This is the focus of the article.

This paper analyze physician-patient relations in health communication from the perspective of interpersonal communication. Interpersonal communication is generally refers to face-to-face communication, being also known as face-to-face communication, person-to-person communication. 
According to one of the modes of communication: the Osgood-Schramm model, medical personnel and patients play the role of communicator and receiver in communication. The doctor disseminate information to patient, the patient is properly accepted, and provide feedback, communication between them is based on the common information space that we all understand.[1] American scholar Burgoon believes that health communication is the interaction between patients and medical providers and interpersonal communication activities in treatment room.[2]

\section{Research Methods}

This study collect first-hand data of doctors and patients through in depth interview and observational method to do exploratory research of doctor-patient communication. The interviewees include doctors and patients, of which 10 doctors are in top three hospitals in Shandong and Sichuan province respectively. Ten patients were interviewed, six patients were carried out in the hospital, four patients have recently had medical experience of general public.

\section{Research Result}

Through the field investigation and interviews with doctors and patients, combined with literature content, the study find that main reason leading to doctor-patient communication obstacle, includes cognitive incongruity and other issues in the process of doctor-patient communication, in addition to external social environment and national policy and other factors. As follows:

\section{Cognitive Incongruity in Doctor-Patient Communication}

\section{Both Doctors and Patients Have Misunderstandings in the Role Definition of Self and Opposite Side}

From the absolute worship of doctors in ancient times to professionalization and marketization of modern doctors, in the envolution of doctor-patient relations, patients' discourse status is rising and the absolute dominance of doctors shows a decreasing trend. But in the current environment, some doctors are too much sure of self-dominance. They gave diagnosis in extremely short time and ignore previous presence of disease symptom, which may cause the doctor to understand patients' illness uncomprehensive and the effect later treatment. For example, the author find out a kind situation in observation of a gynecological hospital in Shandong province. Due to early pregnancy, a woman vomited to syncope, surrounding patients called nurse for her. Ten minutes later, the nurse with a bottle of medicine was ready to put injection. In the whole process, there were no dialogue and interaction between them. From the interpersonal communication model, doctor did not talk clearly about patients' situation they should know to them, and did not collect feedback on disease feelings and views of treatment program from patients and their family members.

But patients and their families will be positioned to come to spend money buying services, being vulnerable groups and consumer. Some patients also believe that medical workers want to make money from them such as receiving envelopes, instead of caring about medical results. These misunderstandings have become a barrier in doctor-patient communication, bringing about distrust between both sides.

\section{The Imbalance of Common Sense Space Caused By Information Imbalance}

If the information passed by doctors and patients is consistent, this common space will exist, the next message can be communicated. Medicine is a subject which has strong technicality and professionality. For patents who did not have relevant background knowledge, it is a little hard to understand. On the one hand, precision of some of media's spread in health communication is not enough, having lots of false medical knowledge, such as" baking soda starved cancer cells" "red meat for carcinogens", resulting in differences between doctors and patients on the understanding of things. On the other hand, the current medical resource have shortages and management mechanism is not perfect. Doctors have many patients to deal with, so they have less time to explain clearly. And some professional knowledge cannot be understood in a short time, so in the selection process 
of doctors, the use of inappropriate language or professional terms may become an obstacle to communication between doctors and patients.

\section{Little Chance of Interaction between Doctors and Patients}

With the continuous development of modern medicine, the state and social investment makes the medical equipment more sophisticated, patients receive more scientific and accurate treatment, at the same time, they have less and less interaction with doctors. And the excessive reliance on modern instruments make doctors ignore full exchange with the patient. Precise medicine age, the increase in medical costs also exacerbated patients' distrust. Aiming at high price of some special medicine, causing expensive medical fees, John Milligan, president of Gillard Scientific, said that:" we will deal with hundreds of organizations." When these drug manufactures ignore the deal with patients, but have a dialogue with doctors mastering prescription and powerful insurance company, problems between patients and medical industry will appear. Not understand drug's R\&D and production are to pay the cost. In addition, in the current domestic medical environment, doctors treat more patients, the time which is balanced to each one is very small. According to a survey of a teriary hospital, health care workers believe that "busy task.no time and energy" is the mainly reason influencing doctor-patient communication, the proportion is up to $87.3 \%$. Therefore, less opportunity for information exchanges have been obstacles between doctors and patients, which in some ways will make the patient produce distrust on doctors slowly, barrying hidden dangers for further doctor-patient dispute.

\section{The Lack of Communication Skills of Health Care Workers}

In communicating with patients, some medical staff did not fully take patients' personal characteristics, gender, family background, religious belief and other factors, only pay attention to disease changes at different stages and corresponding treatment principles. Author interviewed Dr. Zhang, Department of Neurllogy, Sichuan province, he said that in the face of malignant tumors, advanced cancer patients, they often have little communication, the patient himself also understand that, all the disease treatment is to buy a probability. After all, cold fever may be at risk of treatment failure, the tumor is the same. I believe that in the face of the elderly, such word may be able to be accepted, but for young people, doctor still pay attention to language enlightenment and positive incentives, rather than committing fluke mind.

Of course, in the face of some special patients, such as: pregnant women, children the disabled, critically ill persons or patients with sensitive disease, doctors should pay attention to communicate effectively according to patients" own situation. The formation of impression of "clod-blooded" is caused by lack of human quality on medical staff to some extent. Most of today's Chinese medical education is still focusing on teaching knowledge, the ability of students to practice and communicate with patients has not received due attention. In our country, until the fourth year of clinical study and the fifth year of clinical practice, medical students contact with patients, resulting in basic and clinical teaching content not being organically combined, lacking training in contacting and interacting with patients. In current, medical school did not set up systematic communication skills course. Although some institutions will hold relevant lectures, students voluntarily paiticipate in the form [6]

\section{The Negative Emotions of Both Doctors and Patients Due to Their Own Pressure}

In the course of treatment, patients may suffer from double stress, physical and psychological ,coupled with some environmental conditions, family stress, obscure medicine and other unstable factors, patients mood is likely to outbreak on account of small things. The status quo of low income of doctors, heavy workload, overtime and extreme doctor-patient relationship events occurring in reports will give medical staff anxiety, varying degrees of depression and other conditions such as less words. In different character, not want to communicate with people. These above are obstacles for further interaction between doctors and patients. 


\section{Suggested Measures}

In view of the four types of problems above, we propose the countermeasures according to characteristics of interpersonal communication, including two-way communication, strong situationality, timely feedback and diversity of symbol system from the standpoint of communication form, skills, emotional focus and other fields.

\section{Utilize New Technologies to Popularize Medical Knowledge and Innovate Doctor-patient Communication Form}

While treating patients, hospital need to promote medicine knowledge, medical institution can be able to use new media tools and technique to enrich communication means between doctors and patients. For example, a hospital in WuWi used Ipad to contact with critical patients. This tool can be touched, have volume information and creat contents matching therapy. Contents include diet guidance, disease introduction, health education, psychological counseling, family needs in the form of images, audio and video. This approach provide human services for patients by using new technology. Zhongshan hospital in shanghai used VR technique to realize liver transplantation living broadcast, patients can observe operation, which is a bold attempt on doctor-patient communication. It eliminates the mystery of operation itself and operating room, allowing patients to participate by VR, letting them see what the surgery is and what the doctor is doing, which can enhance communication and reduce contradiction between doctors and patients.

\section{Focus on the Patient's Mental and Emotional Exchanges, to Win the Trust of Patients}

Good mental and emotional communication is easy to open patients' heart and build basic trust, promoting following information interaction. Foreign doctors use E4 (engage, empathize, educate, enlist) model to communicate with patients, we can learn from it porbaly. In addition to disease treatment, this model also give patients psychological care and interaction through inquring open questions, making positive and reasonal response to patients, listening for them and effective encouragement.

From production to use of medical supplies, there is complex division of labor, which contains a variety of links. If problems occur in any link, patients' trust will be reduced. In order to win trust from patients, interest-related in medical industry should place patients' interests in a central position, construct health care system guided by patients' value, increase transparency of diagnosis information, help patients to better understand their health status and disease treatment effect.

\section{Paying Attention to the Cultivation of Medical Personnel Communication Ability}

Foreign medical education has focused attention on cultivating communication skills with patients for medical students. We can learn from foreign training model to cultivate enthusiasm of medical students to face to patients, improve medical students' ability to communicate with patients, in order to obtain understanding, trust and respect from patients. American medical colleges attach great importance to foster medical students' communication skills, and request medical educators to explain, teach, evaluate communication skills carefully, letting each doctor not only have superb medical skills, but also have good interaction skills. Status of doctor-patient communication in china only focus on communication in treatment and ignore communication before and after treatment. Today, the network information technology is very developed, microblog, we-chat, video sites and other social media platform can help to extend content and form of doctor-patient communication. A complete doctor-patient communication mode should not be limited to internal communication in hospitals, reasonable and effective communication outside the hospital is an effective way to perfect medical service, improve medical effect and obtain satisfaction and loyalty from patients.

\section{Help Medical Staffs to Resolve Occupational Stress}

In the interview, a 30year-old female patient came to hospital to do the people sugery, according to a doctor's introduction. After family members' signing, doctors performed the operation. However, 
the patient's male partner made trouble in office and carried off medical records. He gave attending female doctor injurious language that you did not deserve to be a doctor. This matter made a deep impression on the doctor. While facing larger work intensity, doctors may be traumatized in the mind. Sometimes the reason is not necessarily caused by external factors, psychological complexity may give doctors big pressure when facing with patients' psychological complexity. Hospital can set up some psychological counseling lectures or build some conpacting room, organize activity of cultural sports to help them relief stress.

In the follow-up study, we can focus on the development of doctor-patient communication model in China, try to design a doctor-patient communication model in line with Chinese medical service situation, develop a different use of doctor-patient communication assessment scale, and study the application of social media in doctor-patient practice to explore effective ways to improve doctorpatient communication quality.

\section{Conclusions}

Through the observation of the two hospitals and interviews with doctors and patients, the article found that although the above content affects the quality of communication between doctors and patients, doctor-patient strategy can be obtained through a conscious training of. Mastering these tactics should follow the time to change, use the latest technology, practice continuously and pay timely attention to new problems and phenomena in the process of doctor-patient communication.

Though from the perspective of interpersonal communication, to examine doctor-patient relationship problems can provide doctor-patient communication with solutions in microcosm. But considering systematically resolving barriers between doctors and patients, we can discover more qualified and effective doctor-patient communication plans in the way of communication mode, system establishment and so on.

\section{Acknowledgement}

The study of scientific information communication and crisis communication from the perspective of risk management in the Department of Humanities and social sciences of the Ministry of Education (16YJC860018)

\section{Reference}

[1] Guo Qinguang .Communication course [M].BeiJing: Renmin university of China press, 1999.P95.

[2] Burgoon."Strangers in a strange land:The Ph.D.in the land of the medical Doctor"[M].Language and Social Psychology,Vol.11,1992.pp.102.

[3] http://international.caixin.com/2017-01-18/101046273.html\#_sinfor=appid:801152585.

[4] FengJunqiang, XiangYanbin.Research on investigative analysis and countermeasures of the doctor-patient communication in a general hospital [J].Chinese health laboratory technology magazine, 2008.18(6): 1199-1200.

[5] YangXinzhe.Resrarch on the influence on doctor-patient relationships of media communication [D].Shanghai: Shanghai Jiaotong University, 2012.6.

[6] SongChunyu, DongHong, WangNan, CuiXiaoguang. Cultivation differences of medical students in America and China [J].China Higher Medical Education, 2012, 10.

[7] YuanWeiwei,XuanJialing. Obstacles and countermeasures of doctor-patient communication in China [J].Medicine and Society, 2013.6.

[8] http://health.sohu.com/20140929/n404744907.shtml. 
[9] Vaughn F Keller, J Gregory Carroll. A New Model for Physician-patient Communication [J].Patient Educ Couns, 1994(23): 131-140.

[10] Zhanghui. Research on improving the ability of doctor-patient communication skills of medical students [J]. Northwest Medical Education, 2006, 14(4): 362.

[11] Liao Chunmei, XuJianxiang. Initial Research on Interns communication skills cultivation. [J]. Journal of Hu Nan Medical College, 2003, 5(2): 44.

[12] Lu Zhongyi. Implemet doctor-patient communication mechanism, improve doctor-patient relationships [J].Chinese Jouanal of hospital Administration, 2002, 18(12):726.

[13] WangJin, Dai Xiaoli. Cultivation and ponder of doctor-patient communication skills of medical students in America [J]. Clinical Education of General Practice, 2005, 3(3): 166.

[14] Wu Caiqin, Li jingjing, Zhu Yunxia. Application of doctor-patient communication skills in offering qualified nursing [J]. Journal of Nursing, 2012.7. 